\title{
How the COVID-19 pandemic changed postoperative infections in urology wards: A retrospective cohort study from two urology departments
}

\author{
Bernardo L. Teixeira ${ }^{1}$; João Cabral ${ }^{1}$; André Marques-Pinto ${ }^{1}$; FernandoVila ${ }^{2}$; \\ Joaquim Lindoro ${ }^{2}$; Avelino Fraga ${ }^{1}$ \\ ${ }^{1}$ Department of Urology, Centro Hospitalar Universitário do Porto, Porto, Portugal; ${ }^{2}$ Department of \\ Urology, Centro Hospitalar do Tâmega e Sousa, Penafiel, Portugal
}

Cite as: Teixeira B, Cabral J, Marques-Pinto A, et al. How the COVID-19 pandemic changed postoperative infections in urology wards: A retrospective cohort study from two urology departments. Can Urol Assoc J 2021 December 21; Epub ahead of print. http://dx.doi.org/10.5489/cuaj.7521

Published online December 21, 2021

Corresponding author: Dr. Bernardo L. Teixeira, Department of Urology, Centro Hospitalar Universitário do Porto, Porto, Portugal; Bernardolat@gmail.com

$* * *$

\section{Abstract}

Introduction: We aimed to compare the rate of postoperative infection and drugresistant organism (DRO) before and during the COVID-19 pandemic in urology departments.

Methods: A retrospective cohort study was carried out. Data from all elective surgical procedures carried out in two urology departments between April and June 2018 and the homologous period in 2020 were collected. Main outcomes were the number of postoperative infections during the pandemic, and the number of DROs. Sample size was calculated based on a 50\% relative reduction of infections during the pandemic. Variables were compared by Chi-squared test, and multivariable logistic regression was used to estimate predictors.

Results: A total of 698 patients undergoing elective surgery were included. The postoperative infection rate during the pre-pandemic period was of $14.1 \%$ compared to $12.1 \%$ during the pandemic $(\mathrm{p}=0.494)$. DROs were lower during the pandemic $(92.3 \%$ vs. $52.4 \%, p=0.002)$. The pandemic period was the main predictor for reduced multidrug-resistant isolates, with an odds ratio of $0.10(\mathrm{p}=0.010,95 \%$ confidence interval 0.016-0.57).

Conclusions: Postoperative infection rates were not significantly reduced during the COVID-19 pandemic, despite the adoption of enhanced infection preventive measures. There was, however, a decrease in the rate of DROs during this period, suggesting a 
secondary benefit to enhanced infection prevention practices adopted during the COVID-19 era.

\section{Introduction}

Hospital acquired infections (HAI) represent a global healthcare crisis, contributing to patient's morbidity, and mortality. [1] According to the European Centre for Disease Prevention and Control (ECDC), HAI represent a significant burden among infectious diseases, and are increasingly caused by multidrug resistant microorganisms [2,3].

In response to the COVID-19 pandemic, hospitals had to reorganize wards, postpone elective procedures, and cancel non-urgent activities. Measures to limit the spread of the disease included restrictions to patient's visitors, avoidance of street clothes by health care professionals, frequent object disinfection and improved hand hygiene, which were all followed in both hospitals included in our analysis [4]. Some of these measures have been shown to lower the rate of hospital acquired infections $[5,6]$. However, little is known about the overall impact of the pandemic on nosocomial infections. Some reports observed an increase in HAI, such as those associated with central lines [7]. Others postulate that while some types of HAI may increase, surgical site infections might be lower as surgical room turnover decreases [8].

We hypothesise that the occurrence of post-operative infection during the pandemic would be lower, particularly in surgical departments, as a consequence of COVID-19 preventive measures. The objective of this study is to compare the rate of post-operative infections and patterns of antimicrobial resistance before and during the pandemic in Urology departments.

\section{Methods}

An observational retrospective cohort study was carried out. Centres participating in this study were the Centro Hospitalar e Universitario do Porto, a university teaching hospital, and Centro Hospitalar Tâmega e Sousa, a secondary care hospital, both localized in the northern region of Portugal. Simulations from Pekar J. et all define the period between October and November 2019 as the plausible interval when the first case of SARS-CoV-2 emerged [9]. As such, data from 2018 was used as the control group, and all elective surgical procedures between April and June 2018 and the homologous period in 2020 (encompassing the peak of the first wave) were collected through patient record consultation. Ambulatory surgical patients have a short exposure time to healthcare facilities, and mainly included those submitted to simple scrotal or penile surgery, with a low rate of complications, and as such were not included in the analysis. Non elective, urgent surgery, such as ureteral stenting or nephrotomy tube placement, was excluded since it comprised patients referred from other emergency departments (for lack of emergent urological care) who were subsequently transferred back to their original hospital, hindering data analysis. 


\section{Definitions and variables}

Patient data included age, sex, level of care (secondary care vs university teaching hospital), post-operative in-hospital length of stay, American Society of

Anaesthesiologist (ASA) score, type of procedure, peri-operative antibiotic prophylaxis, pre-operative urine cultures, peri-operative use of medical devices, post-operative infections, microbial culture (including blood, urine and surgical drain or wound exudate) and antimicrobial susceptibility testing. Patients were considered to have a medical device only when they were admitted or discharged with such devices in place (such as double $\mathrm{J}$ stents, testicular prosthesis, urethral slings, or urinary catheters). Prolonged antibiotic prophylaxis was defined as antibiotic coverage for more than 24 hours after surgery. Standard antibiotic coverage included prophylaxis initiated within 1 hour before surgery and up to 24 hours post-operatively. Post-operative infection was defined as any infection occurring up to 30 days after a surgical procedure in a hospitalized patient, according to the ECDC protocol for surgical site infection and prevention [10]. Infections distant to the surgical site (e.g. catheter associated urinary tract infection or pneumonia after a nephrectomy) were also considered. No distinction between type of infection was made for the analysis. Drug resistant organism (DRO) was defined as a microorganism resistant to at least one agent in one or more classes of antimicrobial categories tested. Culture results labelled as "contaminated" were considered negative. All microorganisms were considered in cases of positive cultures with more than one species. Procedures were grouped as to compare differences at baseline between both periods. Based on baseline risk of infection [11] and surgery type, they were classified as following: Endoscopic intra-renal surgery (Percutaneous nephrolithotomy and retrograde endoscopic intra renal surgery); Ureter and lower urinary tract endoscopic surgery (Transurethral bladder or prostate resection, endoscopic prostatic enucleation, ureter stenosis balloon dilation, ureter stenting, endopyelotomy and semi-rigid urethroscopy; Kidney and ureter surgery (partial and radical nephrectomy, simple nephrectomy, nephroureterectomy, pyeloplasty, renal cyst marsupialization, ureter reimplantation, and adrenalectomy); Prostate surgery (simple and radical prostatectomy); Radical Cystectomy; Genital and reconstructive surgery (circumcision, hydrocelectomy, vasectomy, simple and radical orchiectomy, radical penectomy, urethroplasty, vesico-vaginal fistula correction, urethral slings, surgery for Peyronie's disease and sacral neuromodulation)

\section{Outcomes}

The main outcomes were the number of post-operative infections during the pandemic and the number of drug resistant isolates. We hypothesized that during the pandemic the number of infections would be lower, and that a lower number of DRO would be isolated.

\section{Statistical analysis}

Based on local and European data [12] we estimated a post-operative infection rate of $10 \%$. We postulate a relative reduction of $50 \%$ of post-operative infection during the 
pandemic. Based on these estimates, and for an alfa of $5 \%$ and power of $80 \%$, sample size was calculated to 684 patients.

Data was analysed using IBM SPSS Statistics v.26®. Chi-Square test was used to compare categorial variables between periods. Mann-Whitney U test was used to compare continuous variables, and Fisher's exact test was used to compare culture isolates between both periods. Univariable regression analysis included variables of interest. A stepwise multivariable logistic regression model was developed with $p<0.2$ for the main outcomes $[13,14]$. The model was adjusted for age, sex, ASA score, hospital (level of care), antibiotic prophylaxis, period of observation (pre-, during pandemic), presence of medical devices and pre-operative urine cultures.

Complete case analysis was performed. Odds ratios were reported with $95 \%$ confidence intervals. Statistical significance was considered at an alpha $<0.05$

\section{Results}

Demographic data and main results comparing the pre pandemic and pandemic periods are summarized in Table 1. Final analysis included a total of 698 patients and no patient undergoing elective surgery was excluded. Missing data for variables of interest included information regarding antibiotic prophylaxis for 24 patients (14 during the prepandemic period), and 1 for ASA score. Overall, no significant differences were seen between both periods concerning the type of procedures. The number of radical cystectomies were considerably higher during the pandemic, but the difference was not statistically significant $(\mathrm{p}=0.07)$

The number of patients who did not receive antibiotic prophylaxis was greater during the pandemic ( $4.6 \%$ vs $1.2 \%$; $=0.02$ ). As for the type of antibiotic used, $86.6 \%$, $5.3 \%, 4.8 \%$ and $3.3 \%$ received cefoxitin, cefazolin, ciprofloxacin or other antibiotic before the pandemic, respectively. During the pandemic, antibiotics used remained largely unchanged $(86.1 \%, 6.5 \%, 3 \%$ and $4.3 \%$ for cefoxitin, cefazolin, ciprofloxacin or other antibiotic, respectively; $\mathrm{p}=0.586$ )

The post-operative infection rate during the pre-pandemic period was of $14.1 \%$ comparing to $12.1 \%$ during the pandemic $(\mathrm{p}=0.494)$. No statistically significant difference was observed when analysis was repeated after excluding cases of infection without positive cultures ( $\mathrm{p}=0.356$ ). Only $47.2 \%$ and $62.9 \%$ of cultures were positive in the pre-pandemic and pandemic period, respectively $(\mathrm{p}=0.192)$. Patterns of drug resistance infections were different between groups, with $92.3 \%$ of isolates being categorized as multi drug resistant in the pre-pandemic period compared to $52.4 \%$ during the pandemic $(\mathrm{p}=0.002)$. The pathogens isolated in both periods are presented in Figure 1. Differences between isolated microorganisms were not statistically significant, when tested with Fisher's Exact test ( $\mathrm{p}=0.776)$.

ASA Score was associated with an increase odd of post operative infection (OR $2.09,95 \%$ CI $1.45-3.01 ; \mathrm{p}<0.001)$ and DRO isolates ( $\mathrm{OR}=4.22,95 \% \mathrm{CI} 1.1-15.89$; $\mathrm{p}=0.03$ ) on univariable analysis - Table 2 and Table 3 . On the same analysis, the 
pandemic period was associated with a lower risk of $\mathrm{DRO}(\mathrm{OR}=0.09,95 \%$ CI 0.02 $0.49 ; p=0.005)$, but not with a risk of post operative infection $(p=0.53)$

The stepwise multivariable logistic regression models predicting the number of infections and the isolate's drug resistance pattern are presented in Table 4 and Table 5. The model including the pandemic period and the ASA score was the one best predicting the outcome of DRO isolates, with an OR of $0.1(95 \% \mathrm{CI} 0.07-0.57$; $\mathrm{p}=0.010$ ). Although not statistically significant, a trend towards an increase in the number of post operative infections was observed for the level of care, with an OR of 1.57 (95\% CI $0.97-2.54 ; \mathrm{p}=0.069)$ for the university teaching hospital.

\section{Discussion}

To the best of our knowledge, this is the first paper addressing the differences in postoperative infection patterns during the COVID-19 pandemic on a surgical ward. We have not been able to document a decrease in the number of post-operative infections during the COVID-19 pandemic, but our results show that the number of drug resistant isolates were lower in patients with a nosocomial infection during this period.

According to our results, we believe that hospital preventive measures to limit the spread of the virus contributed in some form to this outcome. Few studies have addressed this issue outside surgical specialties, with conflicting results. Some have documented an increase in colonization or infection with DRO in COVID-19 patients, although analysis was limited to intensive care units, during peak incidence periods and when protective equipment and healthcare professionals were sparse $[15,16]$. Others have documented fewer Clostridium difficile infections and low rates of multi-drug resistant superinfections in COVID-19 patients. [17,18]

Infection prevention include measures such as hand hygiene and room disinfection, antimicrobial stewardship programs, and patient and staff cohorting in cases of DRO infection. Patient cohorting has been referred to as the most effective measure in cases of multi drug resistant infection outbreak [19]. Compliance with infection prevention and control measures has been shown to be heterogenous in different European countries, and several areas have been noted as being critical, namely the number of clinical and infection control staff, and educational programmes [20]. The reduced number of elective surgeries during the pandemic could have had translated in an increase of unoccupied hospital beds and easier patient cohorting in case of DRO bacteria colonization or infection. Yet, this was not the case, as many beds were allocated to COVID-19 or non-surgical patients, even as hospital capacity increased [21]. We argue that the heightened perception by healthcare professionals about the importance of nosocomial infections and about their role in preventing them brought by the COVID-19 pandemic, led to increase adherence to preventive strategies, driving the lower DRO infections number in our sample. Furthermore, increased compliance with best practices for hand hygiene, not only by healthcare staff, but also by patients, could contribute to our findings, as studies have demonstrated high rates of visitor's and 
patient's hand contamination by DRO species, during hospital admission and stay [2224]. Further studies are needed to confirm our hypothesis.

Interestingly, the number of positive cultures was higher during the pandemic in our sample, and this could be the result of increased hand hygiene and adherence to best practice on blood and urine collection, diminishing sample contamination (which were judged as negative in our analysis). However, such differences between periods were not statistically significant. Additionally we observed a non-statistically significant increase in the odd of post operative infections in the university teaching hospital on multivariable regression analysis $(\mathrm{OR}=1.5795 \% \mathrm{CI} 0.97-2.54 ; \mathrm{p}=0.069)$. Nosocomial infections have been showed to increase according to the level of care and hospital size, but as concluded by H. Sax et al, this seems to be partly associated with unfavourable case mix. [25]

Antimicrobial resistances are known to change over time and vary according to region, which could explain our results, independently from the COVID-19 pandemic. However, the annual epidemiological report for 2018 and 2019 from the ECDC addressing antimicrobial resistance in the European Union highlights that more than half of the E. coli and Klebsiella pneumoniae isolates were resistant to at least one antimicrobial group in Portugal, and combined resistance to several antimicrobial groups was frequent. In addition, for most Gram-negative species, changes in resistance patterns between 2015 and 2019 were moderate and remained at high levels. As for Gram-positive bacteria such as Enterococcus species, the most prevalent in our study, a significant decrease in high level gentamicin resistance for $E$. faecalis was noted between both periods ( $31.9 \%$ vs. $26.6 \%$ ), but this was accompanied by an increase in vancomycin resistance in E. faecium isolates, from $10.5 \%$ in 2015 to $18.3 \%$ in 2019 , in the EU. Notably for vancomycin resistant E. faecium, no distinct geographical pattern could be seen, with high levels throughout Europe [26]. Similarly, according to the Canadian antimicrobial resistance surveillance system, the rate of health care associated vancomycin resistant Enterococcus bloodstream infection more than doubled between 2014 and 2018 (from 0.12 to 0.31 cases per 10,000 patient-days) [27]. Additionally invasive E. Coli resistance to third generation cephalosporins is comparable between Portugal and Canada (10-25\%) as reported in both surveillance systems. Our results contradict the increased trend on DRO infection.

Certainly, a factor to be considered if further studies confirm our results, is the inappropriate use of antibiotics in the community, which was showed to be lower during 2020, and could have a major impact on the rate of DRO. Data from INFARMED, the Portuguese drug authority confirms a reduction of $20.7 \%$ ambulatory antibiotic use between January and September 2020 compared to the homologous period in the previous year [28]. Additionally, antibiotic prophylaxis during surgery was lower in our sample (No antibiotic prophylaxis $1.2 \%$ vs $4.6 \%$ in pre pandemic and pandemic period respectively). However, the duration of antibiotic prophylaxis was statistically excluded by our stepwise multivariable regression analysis model. 
As noted by Monnet D. and Harbath S. in their editorial, the COVID-19 pandemic reminds us that compliance with infection preventive measures is critical to ensure the safety of hospitalised patients [29]. The current pandemic has brought dramatic changes to health care, but some silver-linings should be emphasized, and some old lessons relearned. Hospital acquired infections and multi drug resistance microorganism represent a public health crisis that will endure long after the current pandemic, and health care institutions, professionals and patients should do their part to prevent them.

Several limitations to our study can be addressed, mainly related to the retrospective design which makes information bias unavoidable. ECDC protocol was used to define surgical site infection [10] and although the protocol has some objective definitions of infection, it also considers those diagnosed by the attending physician or surgeon. This fact introduces some subjectivity in every day clinical practice that is difficult to overcome, for example when a patient submitted to a partial nephrectomy develops fever with increased analytical inflammatory markers, and negative blood and urine cultures. This clinical scenario could be caused either by infection or be secondary to the surgical trauma or renal ischaemia. Naturally, real infection rates could be either over or underestimated.

Our definition of drug resistance limits comparison to other reports and represents a potential bias. Although the definition of multidrug resistant organisms is not consensual in the literature, efforts have been made to standardize it. The most accepted international terminology defines multidrug resistance (MDR) as nonsusceptibility to at least one agent in three or more antimicrobial categories [30]. We broadened our definition to include resistance to at least one agent in one or more classes, and hence used the terminology "Drug resistant organism" to avoid confusion. We have used such definition as we did not have access to the full antibiogram panel. Nonetheless, we do not believe this to have a major impact in our conclusions as we tried to reinforce how infection preventive measures actively translate into a negative selective pressure of resistant bacteria in a surgical ward (irrespective of the standard definition). Contaminated samples were grouped with negative cultures in order to improve statistical power. This categorization of data represents a potential bias.

Patients admitted to elective surgery during the pandemic might not be comparable to those admitted before the public health care crisis, as resources were concentrated on more serious cases. Multiple endogenous and exogenous risk factors for surgical site infection have been identified, and the former (related to patient's comorbidities), in addition to the type of procedure might be the main predictors of surgical infections [11]. Both endogenous factors (represented by the ASA score) and type of procedure remained mostly unchanged during the studied periods, explaining in part the similar rates of post-operative infections. ASA score is nonetheless an oversimplification of patient's comorbidities, and we have excluded ambulatory and urgent surgery, limiting our analysis and representing a potential selection bias. On the contrary, exogenous risk factors, more dependent on health care staff and infrastructure, 
which changed dramatically during the pandemic, might have a more predominant role on the dissemination of DRO.

Our results were unable to confirm a lower number of post operative infections during the pandemic $(14.1 \%$ vs $12.1 \% ; \mathrm{p}=0.494)$. Sample size calculation was based on a pre-determined relative reduction of $50 \%$ on infection rate between periods. One might argue that we have overestimated the potential difference in the infection rate. However, nosocomial infection rate in Portugal is one of the highest in Europe, doubling those of similar healthcare systems [12,31], and a recent systematic review has reported that $33-55 \%$ of hospital acquired infections might be preventable [32]. Nonetheless, this implies that our study was underpowered to detect a smaller effect size. Additionally, we did not discriminate between different types of infection and this limitation could also justify the negative results in terms of the number of post operative infections, as some types are more dependent on health care factors than others (e.g. Clostridium difficile infection).

\section{Conclusions}

Drug resistant organism isolates were lower during the pandemic in Urology wards. Reinforced infection preventive measures to limit the spread of COVID-19, such as increased hand hygiene, room disinfection and reduced family visits to inpatients could have been responsible for the results. No statistically significant difference was found between the number of post-operative infections in our sample. Further reports, such as those from the ECDC and CDC are needed to confirm our results. Such studies should try to distinguish different types of infections, as they are not homogenously influenced by infection preventive measures applied during the COVID-19 pandemic. Compliance rate with infection preventive measures ought to remain at high levels after the pandemic. 


\section{References}

1. WHO guidelines on hand hygiene in health Care: first global patient safety challenge clean care is safer care. Geneva: World Health Organization; 2009. 3, The burden of health care-associated infection.

2. Cassini A, Plachouras D, Eckmanns T, et al. Burden of six healthcare-associated infections on European population health: estimating incidence-based disabilityadjusted life years through a population prevalence-based modelling study. PLoS Med. 2016;13:1-16.

3. Tacconelli E, Buhl M, Humphreys H, et al. Analysis of the challenges in implementing guidelines to prevent the spread of multidrug-resistant gramnegatives in Europe. BMJ Open. 2019;9:1-8.

4. Nuñez JH, Porcel JA, Pijoan J, et al. Rethinking trauma hospital services in one of Spain's largest university hospitals during the covid-19 pandemic. How can we organize and help? Our experience. Injury. 2020;51:2827-2833

5. Allegranzi B, Pittet D. Role of hand hygiene in healthcare-associated infection prevention. J. Hosp. Infect. Elsevier Ltd; 2009;73:305-315.

6. Banach DB, Bearman GM, Morgan DJ, et al. Infection control precautions for visitors to healthcare facilities. Expert Rev. Anti. Infect. Ther. 2015;13:10471050.

7. Lerose J, Sandhu A, Polistico J, et al. The impact of coronavirus disease 2019 (COVID-19) response on central-line-associated bloodstream infections and blood culture contamination rates at a tertiary-care center in the Greater Detroit area. Infect Control Hosp Epidemiol. 2021;42:997-1000.

8. McMullen KM, Smith BA, Rebmann T. Impact of SARS-CoV-2 on hospital acquired infection rates in the United States: Predictions and early results. Am J Infect Control. 2020;48:1409-1411

9. Pekar J, Worobey M, Moshiri N, et al. Timing the SARS-CoV-2 index case in Hubei province. Science. 2021;372:412-417

10. European centre for disease prevention and control. Surveillance of surgical site infections and prevention indicators in European hospitals - HAI-Net SSI protocol, version 2.2. Stockholm: ECDC; 2017.

11. Grabe M, Botto H, Cek M, et al. Preoperative assessment of the patient and risk factors for infectious complications and tentative classification of surgical field contamination of urological procedures. World J Urol. 2012;30:39-50.

12. Suetens C, Latour K, Kärki T, et al. Prevalence of healthcare-associated infections, estimated incidence and composite antimicrobial resistance index in acute care hospitals and long-term care facilities: Results from two european point prevalence surveys, 2016 to 2017. Eurosurveillance. 2018;23:1-17.

13. Mickey RM, Greenland S. The impact of confounder selection criteria on effect estimation. Am J Epidemiol 1989;129:125-137

14. Bendel RB, Afifi AA. Comparison of stopping rules in forward "stepwise" regression. J Am Stat Assoc 1977;72:46-53.

15. Tiri B, Sensi E, Marsiliani V, et al. Antimicrobial stewardship program, COVID-19, and infection control: Spread of carbapenem-resistant Klebsiella Pneumoniae colonization in ICU COVID-19 patients. What did not work? J Clin Med. 2020;9:2744. 
16. Kampmeier S, Tönnies H, Correa-Martinez CL, et al. A nosocomial cluster of vancomycin resistant Enterococci among COVID-19 patients in an intensive care unit. Antimicrob Resist Infect Control. 2020;9:1-6.

17. Bentivegna E, Alessio G, Spuntarelli V, et al. Impact of COVID-19 prevention measures on risk of health care-associated Clostridium difficile infection. Am J Infect Control. 2021;49:640-642.

18. Garcia-Vidal C, Sanjuan G, Moreno-García E, et al. Incidence of co-infections and superinfections in hospitalized patients with COVID-19: a retrospective cohort study. Clin Microbiol Infect. 2021;27:83-88.

19. Laurent C, Rodriguez-Villalobos H, Rost F, et al. Intensive care unit outbreak of extended-spectrum $\beta$-lactamase-producing Klebsiella Pneumoniae controlled by cohorting patients and reinforcing infection control measures. Infect Control Hosp Epidemiol. 2008;29:517-524.

20. Tacconelli E, Buhl M, Humphreys H, et al. Analysis of the challenges in implementing guidelines to prevent the spread of multidrug-resistant gramnegatives in Europe. BMJ Open. 2019;9:1-8.

21. Vilallonga R, Garcia Ruiz de Gordejuela A, Cossio-Gil Y, et al. Transforming a surgical department during the outbreak of new coronavirus pandemic. Clinical implications. Langenbeck's Arch Surg. 2020;405:867-875.

22. Mody L, Washer LL, Kaye KS, et al. Multidrug-resistant organisms in hospitals: What is on patient hands and in their rooms? Clin Infect Dis. 2019;69:18371844.

23. Landers T, Abusalem S, Coty MB, et al. Patient-centered hand hygiene: The next step in infection prevention. Am. J. Infect. Control. Elsevier Inc; 2012;S11S17.

24. Kim MK, Nam EY, Na SH, et al. Discrepancy in perceptions regarding patient participation in hand hygiene between patients and health care workers. Am J Infect Control. 2015;43:510-515.

25. Sax H, Pittet D. Interhospital Differences in Nosocomial Infection Rates. Arch Intern Med. 2002;162:2437-42.

26. European Centre for Disease Prevention and Control. Antimicrobial resistance in the EU/EEA (EARS-Net) - Annual Epidemiological Report 2019. Stockholm: ECDC; 2020.

27. Public Health Agency of Canada - Canadian Antimicrobial Resistance Surveillance System Report, 2020

28. IFARMED, I.P. Relatório de monitorização de consumo medicamentos em ambulatório 2020 - Available from:

https://www.infarmed.pt/web/infarmed/profissionais-de-saude/utilizacao-edespesa/relatorios/-

/document_library_display/cpjSL7dQXMGI/view/3653922?_110_INSTANCE_ cpjSL7dQX armed $\% 2$ Fprofissionais-de-saude $\% 2$ Futilizacao-e-

despesa\%2Frelatorios\%3Fp_p_id\%3D110_INSTANCE_cpjSL7dQXMGI\%26p _p_lifecycle $\% 3 \mathrm{D} 0 \% 26 \mathrm{p} \_\mathrm{p} \_$state $\% 3$ Dnormal $\% 26 \mathrm{p} \_\mathrm{p} \_$mode $\% 3 \mathrm{Dview} \% 26 \mathrm{p} \_\mathrm{p} \_\mathrm{c}$ ol_id\%3Dcolumn-1\%26p_p_col_pos\%3D1\%26p_p_col_count $\% 3 \mathrm{D} 3$

29. Monnet DL, Harbarth S. Will coronavirus disease (COVID-19) have an impact on antimicrobial resistance? Eurosurveillance. European Centre for Disease Prevention and Control (ECDC); 2020; 1-6. 
30. Magiorakos A, Srinivasan A, Carey RB, et al. Multidrug-resistant, extensively drug-resistant and pandrug-resistant bacteria: an international expert proposal for interim standard definitions for acquired resistance. Clin Microbiol Infect 2011;18:268-81.

31. European Centre for Disease Prevention and Control. Healthcare-associated infections: surgical site infections. In: ECDC. Annual epidemiological report for 2017. Stockholm: ECDC; 2019.

32. Schreiber PW, Sax H, Wolfensberger A, et al. The preventable proportion of healthcare-associated infections 2005-2016: Systematic review and metaanalysis. Infect Control Hosp Epidemiol. 2018;39:1277-1295. 


\section{Figures and Tables}

Fig. 1. Microorganisms isolated in patients with healthcare-associated infections in urology wards according to study period.

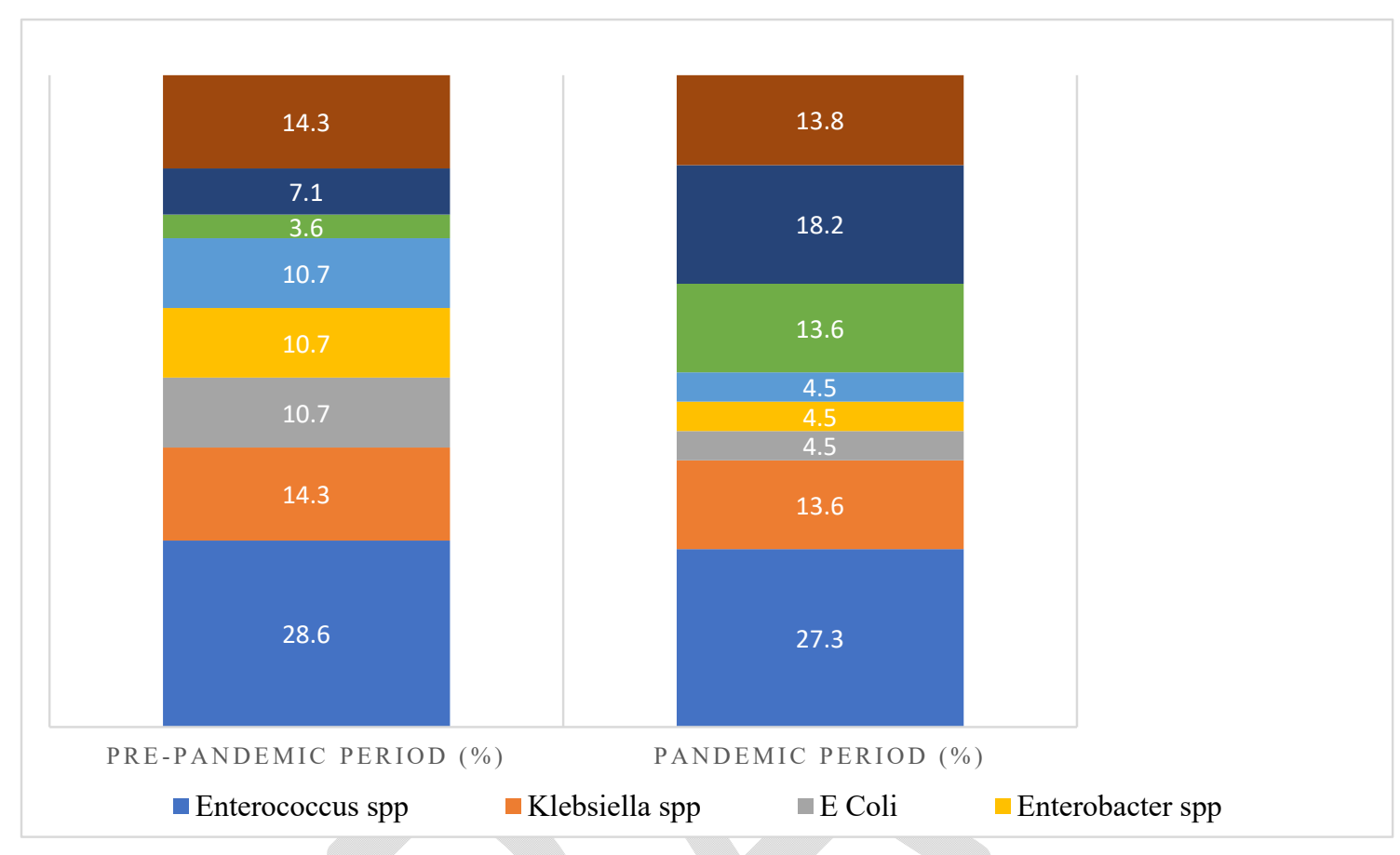




\begin{tabular}{|c|c|c|c|}
\hline & $\begin{array}{l}\text { Pre-pandemic } \\
\text { period } \\
(n=425)\end{array}$ & $\begin{array}{l}\text { Pandemic period } \\
(n=273)\end{array}$ & $\mathbf{p}$ \\
\hline Age, median (IQR) & $65(55-74)$ & $65(54-72)$ & 0.361 \\
\hline Sex, male $(\%)$ & $319(75.1)$ & $203(74.4)$ & 0.858 \\
\hline $\begin{array}{l}\text { Hospital stay, median } \\
\text { (IQR), days }\end{array}$ & $3(2-5)$ & $3(2-6)$ & 0.806 \\
\hline $\operatorname{ASA}(\%)$ & & $2=$ & \\
\hline $\mathrm{I}$ & $29(6.8)$ & $13(4.8)$ & \multirow{4}{*}{0.125} \\
\hline II & $273(64.2)$ & $168(61.5)$ & \\
\hline III & $113(26.6)$ & $90(33)$ & \\
\hline IV & $9(2.1)$ & $2(0.7)$ & \\
\hline Missing & 1 & 0 & \\
\hline $\begin{array}{l}\text { Antibiotic } \\
\text { prophylaxis (\%) }\end{array}$ & & & \\
\hline None & $5(1.2)$ & $12(4.6)$ & \multirow{3}{*}{0.024} \\
\hline Standard & $225(54.7)$ & $139(53.5)$ & \\
\hline Prolonged & $181(44)$ & $109(41.9)$ & \\
\hline Missing & 14 & 13 & \\
\hline Perioperative devices (\%) & $171(40.2)$ & $127(46.7)$ & 0.1 \\
\hline $\begin{array}{l}\text { Preoperative positive urine } \\
\text { culture }(\%)\end{array}$ & $33(24.6)$ & $28(27.5)$ & 0.654 \\
\hline Postoperative infection (\%) & $60(14.1)$ & $33(12.1)$ & 0.494 \\
\hline Positive cultures & $25 / 60(47.2)$ & $22 / 33(62.9)$ & 0.192 \\
\hline Drug-resistance species & $24 / 25(92.3)$ & $11 / 22(52.4)$ & 0.003 \\
\hline $\begin{array}{l}\text { Endoscopic intra-renal } \\
\text { surgery }\end{array}$ & $54(12.7)$ & $33(12.1)$ & 0.816 \\
\hline $\begin{array}{l}\text { Ureter and lower urinary } \\
\text { tract endoscopic surgery }\end{array}$ & $182(42.8)$ & $111(40.7)$ & 0.393 \\
\hline Kidney and ureter surgery & $51(12)$ & $33(12.1)$ & 0.972 \\
\hline Prostate surgery & $74(17.4)$ & $35(12.8)$ & 0.110 \\
\hline Radical cystectomy & $9(2.1)$ & $13(4.8)$ & 0.074 \\
\hline Genital surgery & $55(12.9)$ & $48(17.6)$ & 0.101 \\
\hline
\end{tabular}

ASA: American Society of Anesthesiologists; IQR: interquartile range. 


\begin{tabular}{|l|l|l|}
\hline \multicolumn{2}{|l|}{ Table 2. Univariable regression analysis for risk of postoperative infection } \\
\hline & OR (95\% CI) & p \\
\hline Pandemic period & $0.87(0.55-1.36)$ & 0.53 \\
\hline Medical devices & $1.80(1.16-2.79)$ & 0.008 \\
\hline Male sex & $1.20(0.05-1.25)$ & 0.089 \\
\hline Preoperative urine culture (positive) & $1.96(0.95-4.02)$ & 0.068 \\
\hline Age & $1.01(0.71-2.01)$ & 0.491 \\
\hline ASA score & $2.09(1.45-3.01)$ & $<0.001$ \\
\hline Level of care (tertiary hospital) & $1.59(0.99-2.54)$ & 0.055 \\
\hline $\begin{array}{l}\text { Antibiotic prophylaxis (standard or } \\
\text { prolonged) }\end{array}$ & $2.54(0.33-19.45)$ & 0.37 \\
\hline ASA: Ams & \\
\hline
\end{tabular}

ASA: American Society of Anesthesiologists; CI: confidence interval; OR: odds ratio.

\begin{tabular}{|l|l|l|}
\hline \multicolumn{2}{|l|}{ Table 3. Univariable regression analysis for risks of drug-resistant organism } \\
\hline & OR (95\% CI) & p \\
\hline Pandemic period & $0.09(0.02-0.49)$ & 0.005 \\
\hline Medical devices & $0.24(0.05-1.25)$ & 0.089 \\
\hline Male sex & $1.33(0.28-6.26)$ & 0.716 \\
\hline Preoperative urine culture (positive) & $0.86(0.14-5.2)$ & 0.867 \\
\hline Age & $1.01(0.98-1.06)$ & 0.317 \\
\hline ASA score & $4.22(1.1-15.89)$ & 0.03 \\
\hline Level of care (tertiary hospital) & $0.72(0.16-3.2)$ & 0.675 \\
\hline $\begin{array}{l}\text { Antibiotic prophylaxis (standard or } \\
\text { prolonged) }\end{array}$ & $*$ & $*$ \\
\hline
\end{tabular}

"Univariable analysis was not possible, as only one patient with no antibiotic prophylaxis had a non-drug resistant organism infection, and none had any DRO infection $(\mathrm{p}=1)$. ASA: American Society of Anesthesiologists; CI: confidence interval; OR: odds ratio. 


\begin{tabular}{|l|l|l|}
\hline \multicolumn{2}{|l|}{ Table 4. Multivariate regression analysis for risks of postoperative infection } \\
\hline & OR (95\% CI) & p \\
\hline Hospital (level of care) & $1.57(0.97-2.54)$ & 0.069 \\
\hline Pandemic period & $0.84(0.53-1.34)$ & 0.469 \\
\hline ASA score & & \\
\hline II & $1.03(0.49-5.62)$ & 0.418 \\
\hline III & $3.72(1.08-12.84)$ & 0.037 \\
\hline IV & $14.81(2.70-81.11)$ & 0.002 \\
\hline Presence of medical devices & $2.17(1.37-3.43)$ & 0.001 \\
\hline
\end{tabular}

ASA: American Society of Anesthesiologists; CI: confidence interval; OR: odds ratio.

\begin{tabular}{|l|l|l|}
\hline \multicolumn{3}{|l|}{ Table 5. Stepwise multivariate regression analysis for risks of drug-resistant organism isolates } \\
\hline & OR (95\% CI) & p \\
\hline Period & $0.1(0.07-0.57)$ & 0.010 \\
\hline ASA score & & \\
\hline III & & 0.072 \\
\hline
\end{tabular}

${ }^{a}$ ASA score of 4 was excluded as all patients $(n=3)$ had DRO isolates. ASA: American Society of Anesthesiologists; CI: confidence interval; OR: odds ratio. 